\title{
HOMOTOPY GROUPS OF SPHERES AND LIPSCHITZ HOMOTOPY GROUPS OF HEISENBERG GROUPS
}

\author{
PIOTR HAJŁASZ, ARMIN SCHIKORRA, JEREMY T. TYSON
}

\begin{abstract}
We provide a sufficient condition for the nontriviality of the Lipschitz homotopy group of the Heisenberg group, $\pi_{m}^{\mathrm{Lip}}\left(\mathbb{H}_{n}\right)$, in terms of properties of the classical homotopy group of the sphere, $\pi_{m}\left(\mathbb{S}^{n}\right)$. As an application we provide a new simplified proof of the fact that $\pi_{n}^{\operatorname{Lip}}\left(\mathbb{H}_{n}\right) \neq\{0\}, n=1,2, \ldots$, and we prove a new result that $\pi_{4 n-1}^{\mathrm{Lip}}\left(\mathbb{H}_{2 n}\right) \neq\{0\}$ for $n=1,2, \ldots$ The last result is based on a new generalization of the Hopf invariant. We also prove that Lipschitz mappings are not dense in the Sobolev space $W^{1, p}\left(\mathcal{M}, \mathbb{H}_{2 n}\right)$ when $\operatorname{dim} \mathcal{M} \geq 4 n$ and $4 n-1 \leq p<4 n$.
\end{abstract}

\section{INTRODUCTION}

In this paper, we provide further evidence for the role of Lipschitz homotopy groups in the development of analysis on (non-Riemannian) metric spaces, and specifically, in the study of Sobolev mappings with non-Riemannian target spaces such as the sub-Riemannian Heisenberg group. We link the study of Lipschitz homotopy groups of Heisenberg groups with classical homotopy theory through a new notion of rankessential homotopy groups (Definition 1.4). Using this approach, we provide new and simplified proofs of the nontriviality of certain Lipschitz homotopy groups of Heisenberg groups (previously established in [1) as well as new examples of nontrivial Lipschitz homotopy groups. These results have applications to the problem of density of Lipschitz mappings in Sobolev spaces with Heisenberg targets.

The Heisenberg group $\mathbb{H}_{n}$ is $\mathbb{R}^{2 n+1}$ equipped with the so called Carnot-Carathéodory metric $d_{c c}$. For every compact set $K$ there is a constant $C \geq 1$ such that $C^{-1}|x-y| \leq d_{c c}(x, y) \leq C|x-y|^{1 / 2}$ for $x, y \in K$. Thus $\mathbb{H}_{n}$ is homeomorphic to $\mathbb{R}^{2 n+1}$ and the identity mapping

2010 Mathematics Subject Classification. Primary 53C17; Secondary 46E35, 55Q40, 55Q25.

P.H. was supported by NSF grant DMS-1161425. A.S. was supported by DAAD fellowship D/12/40670. J.T.T. was supported by NSF grant DMS-1201875. 
id $: \mathbb{H}_{n} \rightarrow \mathbb{R}^{2 n+1}$ is locally Lipschitz. However, the inverse mapping id $: \mathbb{R}^{2 n+1} \rightarrow \mathbb{H}_{n}$ is only locally Hölder continuous with exponent $1 / 2$. There is no bi-Lipschitz homeomorphism between $\mathbb{H}_{n}$ and $\mathbb{R}^{2 n+1}$, because the Hausdorff dimension of every open set in $\mathbb{H}_{n}$ is $2 n+2$. The following result is well known.

Proposition 1.1. If $f: \mathbb{R}^{k} \supset \Omega \rightarrow \mathbb{H}_{n}$ is Lipschitz continuous, where $\Omega$ is open, then it is locally Lipschitz continuous as a mapping into $\mathbb{R}^{2 n+1}$. Hence $f$ is differentiable a.e. Moreover, rank $d f \leq n$ a.e.

Since $\mathbb{H}_{n}$ is homeomorphic to $\mathbb{R}^{2 n+1}$, all of its homotopy groups are trivial. On the other hand the Heisenberg group, as an object of study from the viewpoint of geometric analysis on metric spaces, is naturally equipped with its Carnot-Carathéodory metric $d_{c c}$ (or other metrics biLipschitz equivalent to $d_{c c}$ ). As observed above, the Euclidean metric is not of this type. In the framework of analysis on metric spaces it is natural to consider Lipschitz homotopy groups, which are only insensitive to bi-Lipschitz deformation. The Lipschitz homotopy groups $\pi_{n}^{\mathrm{Lip}}(X)$ of a metric space $X$ are defined in the same way as the classical homotopy groups with the difference that now both mappings and homotopies between them are required to be Lipschitz.

In the case of Riemannian manifolds homotopy groups and Lipschitz homotopy groups are the same since continuous mappings can be smoothly approximated. However for non-smooth spaces they may differ. The Heisenberg group is an example since its $n$th Lipschitz homotopy group $\pi_{n}^{\mathrm{Lip}}\left(\mathbb{H}_{n}\right)$ is non-trivial, [1]. However, $\pi_{m}^{\mathrm{Lip}}\left(\mathbb{H}_{n}\right)=\{0\}$ for all $1 \leq m<n$, [27], and $\pi_{m}^{\operatorname{Lip}}\left(\mathbb{H}_{1}\right)=\{0\}$ for all $m \geq 2$, [28]. The results from [1, 27] stated here did not use the language of Lipschitz homotopy groups, but they were translated into that language in [7]. These results show an analogy between the Lipschitz homotopy groups of $\mathbb{H}_{n}$ and the homotopy groups of the sphere $\mathbb{S}^{n}$. The nontriviality of $\pi_{n}^{\mathrm{Lip}}\left(\mathbb{H}_{n}\right)$ is based on the following fact (see [1, Section 4], [7, Theorem 3.2], [8, Example 3.1]).

Proposition 1.2. There is a bi-Lipschitz embedding $\phi: \mathbb{S}^{n} \rightarrow \mathbb{H}_{n}$ of the sphere $\mathbb{S}^{n}$ which is smooth as a mapping to $\mathbb{R}^{2 n+1}$.

It was proved in [1] (see also [7]) that such an embedding cannot be extended to a Lipschitz map $\Phi: \mathbb{B}^{n+1} \rightarrow \mathbb{H}_{n}$. Another simpler proof of this fact is provided below. See the proof that $\pi_{n}\left(\mathbb{S}^{n}\right)$ is rank-essential later in this section. Thus $\pi_{n}^{\operatorname{Lip}}\left(\mathbb{H}_{n}\right) \neq\{0\}$. To emphasize the analogy between $\pi_{m}\left(\mathbb{S}^{n}\right)$ and $\pi_{m}^{\mathrm{Lip}}\left(\mathbb{H}_{n}\right)$ it was asked in [7, Question 4.16] whether 
any bi-Lipschitz embedding $\phi: \mathbb{S}^{n} \rightarrow \mathbb{H}_{n}$ induces an injective homomorphism $\pi_{m}\left(\mathbb{S}^{n}\right) \rightarrow \pi_{m}^{\operatorname{Lip}}\left(\mathbb{H}_{n}\right)$. Actually the authors of the question expected that if a smooth map $f: \mathbb{S}^{m} \rightarrow \mathbb{S}^{n}$ is not homotopic to a constant map, $0 \neq[f] \in \pi_{m}\left(\mathbb{S}^{n}\right)$, then the map $g=\phi \circ f: \mathbb{S}^{m} \rightarrow \mathbb{H}_{n}$ cannot be extended to a Lipschitz map $G: \mathbb{B}^{m+1} \rightarrow \mathbb{H}_{n}$. As will be explained below there were strong reasons based on the Sard theorem to believe in this conjecture, but surprisingly the conjecture is false! Recently, Wenger and Young [28, Theorem 1] proved the following result.

Theorem 1.3. If $\alpha: \mathbb{S}^{n} \rightarrow \mathbb{H}_{n}$ and $\beta: \mathbb{S}^{m} \rightarrow \mathbb{S}^{n}$ are Lipschitz and $n+2 \leq m<2 n-1$, then the map $g=\alpha \circ \beta: \mathbb{S}^{m} \rightarrow \mathbb{H}_{n}$ can be extended to a Lipschitz map $G: \mathbb{B}^{m+1} \rightarrow \mathbb{H}_{n}$.

In particular $\pi_{7}\left(\mathbb{S}^{5}\right)=\mathbb{Z}_{2}$, so there is a smooth map $f: \mathbb{S}^{7} \rightarrow \mathbb{S}^{5}$ that is not homotopic to a constant map, but if $\phi: \mathbb{S}^{5} \rightarrow \mathbb{H}_{5}$ is a biLipschitz embedding, then $g=\phi \circ f: \mathbb{S}^{7} \rightarrow \mathbb{H}_{5}$ has a Lipschitz extension $G: \mathbb{B}^{8} \rightarrow \mathbb{H}_{5}$. This is just one example, but the above theorem leads to many more examples. It just suffices to look at the table of the homotopy groups of the spheres to find cases when $\pi_{m}\left(\mathbb{S}^{n}\right) \neq\{0\}$ and $n+2 \leq m<2 n-1$. It is important to note here that it does not necessarily imply that $\pi_{m}^{\mathrm{Lip}}\left(\mathbb{H}_{n}\right)=\{0\}$, because in this construction we consider mappings to $\mathbb{H}_{n}$ that factor through $\mathbb{S}^{n}$ via a bi-Lipschitz embedding into $\mathbb{H}_{n}$. Perhaps there are other mappings from $\mathbb{S}^{m}$ to $\mathbb{H}_{n}$ that are not Lipschitz homotopic to constant mappings.

Definition 1.4. We say that the homotopy group $\pi_{m}\left(\mathbb{S}^{n}\right)$ is rankessential if there is $f \in C^{\infty}\left(\mathbb{S}^{m}, \mathbb{S}^{n}\right)$ with the following property $(\mathrm{R})$ : for every Lipschitz extension $F: \mathbb{B}^{m+1} \rightarrow \mathbb{R}^{n+1},\left.F\right|_{\partial \mathbb{B}^{m+1}}=f$, we have

$$
\operatorname{rank} d F=n+1
$$

on a set of positive measure.

Clearly if $\pi_{m}\left(\mathbb{S}^{n}\right)$ is rank-essential, then $\pi_{m}\left(\mathbb{S}^{n}\right) \neq\{0\}$. The definition is motivated by the following result.

Theorem 1.5. If $\pi_{m}\left(\mathbb{S}^{n}\right)$ is rank-essential, then $\pi_{m}^{\mathrm{Lip}}\left(\mathbb{H}_{n}\right) \neq\{0\}$.

Proof. Suppose to the contrary that $\pi_{m}\left(\mathbb{S}^{n}\right)$ is rank-essential and that $\pi_{m}^{\operatorname{Lip}}\left(\mathbb{H}_{n}\right)=\{0\}$. Let $f \in C^{\infty}\left(\mathbb{S}^{m}, \mathbb{S}^{n}\right)$ be a mapping with property $(\mathrm{R})$. Since $\pi_{m}^{\operatorname{Lip}}\left(\mathbb{H}_{n}\right)=\{0\}, g=\phi \circ f: \mathbb{S}^{m} \rightarrow \mathbb{H}_{n}$ has a Lipschitz extension $G: \mathbb{B}^{m+1} \rightarrow \mathbb{H}_{n}$. Here $\phi: \mathbb{S}^{n} \rightarrow \mathbb{H}_{n}$ is a bi-Lipschitz embedding from Proposition 1.2. By Proposition 1.1 $\operatorname{rank} d G \leq n$ a.e., where now we regard $G$ as a mapping into $\mathbb{R}^{2 n+1}$. The mapping $\phi^{-1}: \phi\left(\mathbb{S}^{n}\right) \rightarrow \mathbb{S}^{n} \subset$ 
$\mathbb{R}^{n+1}$ is smooth and hence admits a smooth extension $\Psi: \mathbb{R}^{2 n+1} \rightarrow$ $\mathbb{R}^{n+1}$. Clearly $F=\Psi \circ G: \mathbb{B}^{m+1} \rightarrow \mathbb{R}^{n+1}$ is a Lipschitz extension of $\left.\Psi \circ G\right|_{\partial \mathbb{B}^{m+1}}=f$. Since $\operatorname{rank} d G \leq n$ a.e., we conclude that rank $d F=$ $\operatorname{rank} d(\Psi \circ G) \leq n$, which contradicts property $(\mathrm{R})$ of $f$.

From Theorem 1.3 and the proof of Theorem 1.5 we obtain the following corollary. (See [28, Theorem 2] for a stronger statement where it is shown that the corollary is true also for $m=n+1$.)

Corollary 1.6. If $n+2 \leq m<2 n-1$, then $\pi_{m}\left(\mathbb{S}^{n}\right)$ is not rankessential.

In particular if $n+2 \leq m<2 n-1$ and $\pi_{m}\left(\mathbb{S}^{n}\right) \neq\{0\}$ (for example $\left.\pi_{7}\left(\mathbb{S}^{5}\right)=\mathbb{Z}_{2}\right)$, then every smooth mapping $f: \mathbb{S}^{m} \rightarrow \mathbb{S}^{n}$ such that $[f] \neq$ 0 admits a Lipschitz extension $F: \mathbb{B}^{m+1} \rightarrow \mathbb{R}^{n+1}$ with rank $d F \leq n$ a.e., despite the fact that the image of $F$ contains the unit $(n+1)$ dimensional ball. Indeed, otherwise we could pick a point in $\mathbb{B}^{n+1}$ $F\left(\mathbb{B}^{n+1}\right)$ and retract $F$ onto $\mathbb{S}^{n}$.

The main result of the paper reads as follows.

Theorem 1.7. The homotopy groups $\pi_{n}\left(\mathbb{S}^{n}\right)$ and $\pi_{4 n-1}\left(\mathbb{S}^{2 n}\right)$ are rank-essential for $n=1,2,3, \ldots$ and hence $\pi_{n}^{\operatorname{Lip}}\left(\mathbb{H}_{n}\right) \neq\{0\}$ and $\pi_{4 n-1}^{\mathrm{Lip}}\left(\mathbb{H}_{2 n}\right) \neq\{0\}$.

According to the Serre finiteness theorem [25] these are the only infinite homotopy groups of spheres. The proof of Theorem 1.7 is based on differential forms. It is done explicitly in the case of $\pi_{4 n-1}^{\mathrm{Lip}}\left(\mathbb{H}_{2 n}\right)$ and implicitly in the case of $\pi_{n}^{\mathrm{Lip}}\left(\mathbb{H}_{n}\right)$. In the latter case we use the fact that the ball cannot be retracted to the boundary which can be easily proved with the help of differential forms. The language of differential forms is useful when one wants to detect the rational homotopy groups of $C W$ complexes $\pi_{m}(X) \otimes \mathbb{Q}$. This is the so-called rational homotopy theory discovered by Sullivan [11. However in the case of spheres the rational homotopy groups $\pi_{m}\left(\mathbb{S}^{n}\right) \otimes \mathbb{Q}$ are nontrivial exactly in the cases covered by Theorem 1.7. This follows from the Serre finiteness theorem. It would be very interesting to see if $\pi_{m}\left(\mathbb{S}^{n}\right)$ is rank-essential for other values of $m$ and $n$.

The fact that $\pi_{n}^{\operatorname{Lip}}\left(\mathbb{H}_{n}\right) \neq\{0\}$ was proved in [1], but the proof presented here is different and simpler since it does not refer to pure unrectifiability of the Heisenberg group, neither to the degree theory. Another proof of an even more general result that also does not employ pure unrectifiability was given in [23]. 
Proof that $\pi_{n}\left(\mathbb{S}^{n}\right)$ is rank-essential. Let $f: \mathbb{S}^{n} \rightarrow \mathbb{S}^{n}$ be the identity map. If $F: \mathbb{B}^{n+1} \rightarrow \mathbb{R}^{n+1}$ is a Lipschitz extension, then $\mathbb{B}^{n+1} \subset$ $F\left(\mathbb{B}^{n+1}\right)$. In particular the image of $F$ has positive $(n+1)$-dimensional measure. On the other hand for Lipschitz mappings we have ([10, Theorem 3.2.3])

$$
\left|F\left(\mathbb{B}^{n+1}\right)\right| \leq \int_{\mathbb{B}^{n+1}}|\operatorname{det} d F| .
$$

Since $\left|F\left(\mathbb{B}^{n+1}\right)\right|>0$ we deduce that rank $d F \geq n+1$ on a set of positive measure. Thus $\pi_{n}\left(\mathbb{S}^{n}\right)$ is rank-essential and hence $\pi_{n}^{\mathrm{Lip}}\left(\mathbb{H}_{n}\right) \neq\{0\}$.

In the last step of the proof we could refer to the Sard theorem for Lipschitz mappings [22, Theorem 7.6] instead of the integral inequality used above. Assuming rank $d F \leq n$ a.e. we would conclude that all points in $\mathbb{B}^{n+1}$ are critical and hence $\left|F\left(\mathbb{B}^{n+1}\right)\right|=0$.

We now investigate the connection to the Sard theorem in greater detail.

Proposition 1.8. Let $f \in C^{\infty}\left(\mathbb{S}^{m}, \mathbb{S}^{n}\right), m \geq n, 0 \neq[f] \in \pi_{m}\left(\mathbb{S}^{n}\right)$. Let

$$
F: \mathbb{B}^{m+1} \rightarrow \mathbb{R}^{n+1},\left.\quad F\right|_{\partial \mathbb{B}^{m+1}}=f,
$$

be of class $C^{k, 1}, k \geq \max \{m-n, 1\}$. Then rank $d F=n+1$ on an open set.

Here, by $C^{k, 1}$ we denote the class of $C^{k}$ functions whose $k$ th order derivatives are Lipschitz continuous.

Indeed, if rank $d F \leq n$ everywhere, then all points in $\mathbb{B}^{m+1}$ are critical and according to the Sard theorem, [2], the measure of the set

$$
\mathbb{B}^{n+1} \subset F\left(\mathbb{B}^{m+1}\right)=F(\text { Crit } F)
$$

equals zero which is a clear contradiction.

In view of the above discussion it would be natural to expect that if $0 \neq[f] \in \pi_{m}\left(\mathbb{S}^{n}\right)$ then any Lipschitz extension $F$ should satisfy $\operatorname{rank} d F=n+1$ on a set of positive measure. However, the result of Wenger and Young [28, Theorem 2] shows that this is not always the case, see Corollary 1.6. Their proof employs an argument of Kaufman [21], who constructed a surprising example of a surjective mapping $F \in C^{1}\left(\mathbb{R}^{n+1}, \mathbb{R}^{n}\right)$ with $\operatorname{rank} d F \leq 1$ everywhere.

One motivation for studying Lipschitz homotopy groups stems from the problem of approximation of Sobolev mappings. In the classical 
setting the answer to the question whether smooth or equivalently Lipschitz mappings $\operatorname{Lip}(\mathcal{M}, \mathcal{N})$ between compact Riemannian manifolds are dense in the Sobolev space of mappings $W^{1, p}(\mathcal{M}, \mathcal{N})$ heavily depends on the homotopy groups of $\mathcal{N}$, see [3, 4, 12, 13, 15, 18]. Here $\mathcal{M}$ may have boundary, but $\partial \mathcal{N}=\emptyset$. More precisely, if $1 \leq p<\operatorname{dim} \mathcal{M}$ and $\pi_{[p]}(\mathcal{N}) \neq\{0\}$, where $[p]$ is the integral part of $p$, then Lipschitz maps are not dense in $W^{1, p}(\mathcal{M}, \mathcal{N})$. In the case of Sobolev mappings into the Heisenberg group it appears that the density of Lipschitz mappings $\operatorname{Lip}\left(\mathcal{M}, \mathbb{H}_{n}\right)$ in $W^{1, p}\left(\mathcal{M}, \mathbb{H}_{n}\right)$, where $\mathcal{M}$ is a compact Riemannian manifold with or without boundary, depends on Lipschitz homotopy groups of $\mathbb{H}_{n}$. For example it was proven in [7] that if $\operatorname{dim} \mathcal{M} \geq n+1$ and $n \leq p<n+1$, then Lipschitz maps $\operatorname{Lip}\left(\mathcal{M}, \mathbb{H}_{n}\right)$ are not dense in $W^{1, p}\left(\mathcal{M}, \mathbb{H}_{n}\right)$. Note that in this case $\pi_{[p]}^{\operatorname{Lip}}\left(\mathbb{H}_{n}\right)=\pi_{n}^{\text {Lip }}\left(\mathbb{H}_{n}\right) \neq\{0\}$. In this paper we extend this result as follows.

Theorem 1.9. If $\mathcal{M}$ is a compact Riemannian manifold with or without boundary of dimension $\operatorname{dim} \mathcal{M} \geq 4 n$, then Lipschitz mappings $\operatorname{Lip}\left(\mathcal{M}, \mathbb{H}_{2 n}\right)$ are not dense in $W^{1, p}\left(\mathcal{M}, \mathbb{H}_{2 n}\right)$ when $4 n-1 \leq p<4 n$.

Again, according to Theorem [1.7, $\pi_{[p]}^{\mathrm{Lip}}\left(\mathbb{H}_{2 n}\right)=\pi_{4 n-1}^{\mathrm{Lip}}\left(\mathbb{H}_{2 n}\right) \neq\{0\}$. On the other hand we would like to point out that it is possible to construct a smooth manifold $\mathcal{N}$ with one point singularity such that all its Lipschitz homotopy groups are trivial, yet Lipschitz mappings into $\mathcal{N}$ are not dense in the space of Sobolev mappings into $\mathcal{N}$, see [17].

The paper is organized as follows. In Section 2 we provide a brief introduction to the Heisenberg group and we prove Proposition 1.1. This proof is well known, but we recall it here for the sake of completeness and to see how the language of differential forms and their weak exterior derivatives can be used. Such an approach is an essential ingredient in this paper. In Section 3 we briefly recall the definition of Sobolev mappings into $\mathbb{H}_{n}$. In Section 4 we collect basic results about differential forms, DeRham cohomology and Sobolev spaces. We use these facts to generalize in Section 5 the Hopf invariant to Lipschitz mappings into Euclidean spaces whose derivative has low rank. SUch generalization is essential for the proof of Theorem [1.7] which is done in Section 6. Finally, in Section 7 we prove Theorem 1.9.

Those who are interested in the generalized Hopf invariant and its applications to homotopy groups of spheres may skip Sections 2 and 3 and read Sections 4.6. This material is of independent interest and does not involve Heisenberg groups. 
Acknowledgments: We thank the reviewer for the careful reading of our manuscript and the valuable comments.

\section{The Heisenberg Groups}

The Heisenberg group is a Lie group $\mathbb{H}_{n}=\mathbb{C}^{n} \times \mathbb{R}=\mathbb{R}^{2 n+1}$ equipped with the group law

$$
(z, t) *\left(z^{\prime}, t^{\prime}\right)=\left(z+z^{\prime}, t+t^{\prime}+2 \operatorname{Im}\left(\sum_{j=1}^{n} z_{j} \overline{z_{j}^{\prime}}\right)\right) .
$$

A basis of left invariant vector fields is given by

$$
X_{j}=\frac{\partial}{\partial x_{j}}+2 y_{j} \frac{\partial}{\partial t}, Y_{j}=\frac{\partial}{\partial y_{j}}-2 x_{j} \frac{\partial}{\partial t}, j=1, \ldots, n, \text { and } T=\frac{\partial}{\partial t} .
$$

Here and in what follows we use the notation

$$
(z, t)=\left(z_{1}, \ldots, z_{n}, t\right)=\left(x_{1}, y_{1}, \ldots, x_{n}, y_{n}, t\right) .
$$

The Heisenberg group is equipped with the horizontal distribution $H \mathbb{H}_{n}$, which is defined at every point $p \in \mathbb{H}_{n}$ by

$$
H_{p} \mathbb{H}_{n}=\operatorname{span}\left\{X_{1}(p), Y_{1}(p), \ldots, Y_{1}(p), Y_{n}(p)\right\} .
$$

The distribution $H \mathbb{H}_{n}$ is equipped with the left invariant metric $\mathbf{g}$ such that the vectors $X_{1}(p), Y_{1}(p), \ldots, X_{n}(p), Y_{n}(p)$ are orthonormal at every point $p \in \mathbb{H}_{n}$. An absolutely continuous curve $\gamma:[a, b] \rightarrow \mathbb{H}_{n}$ is called horizontal if $\gamma^{\prime}(s) \in H_{\gamma(s)} \mathbb{H}_{n}$ for almost every $s$. The Heisenberg group $\mathbb{H}_{n}$ is equipped with the Carnot-Carathéodory metric $d_{c c}$ which is defined as the infimum of the lengths of horizontal curves connecting two given points. The length of the curve is computed with respect to the metric $\mathbf{g}$ on $H \mathbb{H}_{n}$. It is well known that any two points in $\mathbb{H}_{n}$ can be connected by a horizontal curve and hence $d_{c c}$ is a true metric. Actually, $d_{c c}$ is topologically equivalent to the Euclidean metric. Moreover, for any compact set $K$ there is a constant $C \geq 1$ such that

$$
C^{-1}|p-q| \leq d_{c c}(p, q) \leq C|p-q|^{1 / 2}
$$

for all $p, q \in K$. In what follows $\mathbb{H}_{n}$ will always be regarded as the metric space $\left(\mathbb{H}_{n}, d_{c c}\right)$. It follows from (2.1) that the identity mapping from $\mathbb{H}_{n}$ to $\mathbb{R}^{2 n+1}$ is locally Lipschitz, but its inverse is only locally Hölder continuous with exponent $1 / 2$. The Hausdorff dimension of any open set in $\mathbb{H}_{n}$ equals $2 n+2$ and hence $\mathbb{H}_{n}$ is not bi-Lipschitz homeomorphic to $\mathbb{R}^{2 n+1}$, not even locally. 
Proof of Proposition 1.1. If $f=\left(f_{1}, g_{1}, \ldots, f_{n}, g_{n}, h\right): \Omega \rightarrow \mathbb{H}_{n}$ is a Lipschitz mapping from a domain $\Omega \subset \mathbb{R}^{k}$, then it is locally Lipschitz as a mapping into $\mathbb{R}^{2 n+1}$ and hence is differentiable a.e. It follows that the derivative of $f$ is horizontal, i.e. $d f(p)$ maps the tangent space $T_{p} \mathbb{R}^{k}$ into the horizontal space $H_{f(p)} \mathbb{H}_{n} \subset T_{f(p)} \mathbb{R}^{2 n+1}$. Indeed, $f$ maps straight lines into Lipschitz curves, and Lipschitz curves in $\mathbb{H}_{n}$ are horizontal, [16, Proposition 11.4]. Thus $d f$ maps vectors tangent to straight lines into vectors tangent to horizontal curves. Hence $d f(p)\left(T_{p} \mathbb{R}^{k}\right) \subset H_{f(p)} \mathbb{H}_{n}$ for a.e. $p \in \mathbb{R}^{k}$. Let

$$
\alpha=d t+2 \sum_{j}\left(x_{j} d y_{j}-y_{j} d x_{j}\right)
$$

be the standard contact form on $\mathbb{R}^{2 n+1}$. It is easy to see that the kernel of $\alpha(p), p \in \mathbb{R}^{2 n+1}$, i.e. the collection of vectors $v$ such that $\alpha(p) v=0$, coincides with the horizontal space $H_{p} \mathbb{H}_{n}$. Hence horizontality of the derivative of $f$ means that $f^{*} \alpha(p)=0$ for a.e. $p$, i.e.

$$
d h+2 \sum_{j=1}^{n}\left(f_{j} d g_{j}-g_{j} d f_{j}\right)=0 \quad \text { a.e. }
$$

Since the functions are Lipschitz continuous we can take the distributional exterior derivative (see Lemma 4.1), obtaining

$$
\sum_{j=1}^{n} d f_{j} \wedge d g_{j}=0
$$

In other words if $\omega=\sum_{j} d x_{j} \wedge d y_{j}$ is a symplectic form on $\mathbb{R}^{2 n}$ and $F=\left(f_{1}, g_{1}, \ldots, f_{n}, g_{n}\right)$ is a composition of $f$ with the projection onto $\mathbb{R}^{2 n}$, then $F^{*} \omega=0$ a.e. as a pointwise equality. Let $\mathcal{J}: T_{q} \mathbb{R}^{2 n} \rightarrow T_{q} \mathbb{R}^{2 n}$ be given by

$$
\mathcal{J}\left(\sum_{j=1}^{n}\left(a_{j} \frac{\partial}{\partial x_{j}}+b_{j} \frac{\partial}{\partial y_{j}}\right)\right)=\sum_{j=1}^{n}\left(-b_{j} \frac{\partial}{\partial x_{j}}+a_{j} \frac{\partial}{\partial y_{j}}\right) .
$$

Then for any vectors $u, v \in T_{q} \mathbb{R}^{2 n}$ we have $\omega(q)(u, v)=-\langle u, \mathcal{J} v\rangle$. If $f$ is differentiable at a point $p \in \mathbb{R}^{k}$ and $\left(F^{*} \omega\right)(p)=0$, then for any vectors $u, v \in V:=d F(p)\left(T_{p} \mathbb{R}^{k}\right) \subset T_{F(p)} \mathbb{R}^{2 n}$ we have

$$
\omega(F(p))(u, v)=-\langle u, \mathcal{J} v\rangle=0 .
$$

Thus the space $V$ is orthogonal to $\mathcal{J} V$ and hence $\operatorname{dim} V \leq n$. The rows of the matrix $d f$ are $\nabla f_{1}, \nabla g_{1}, \ldots, \nabla f_{n}, \nabla g_{n}, \nabla h$. We proved that the rank of the minor formed by the first $2 n$ rows is at most $n$. According to (2.3) the last row is linearly dependent on the first $2 n$ rows and hence rank $d f \leq n$ a.e. The proof is complete. 


\section{Sobolev Mappings into $\mathbb{H}_{n}$}

In this section we briefly recall the definition of the space of Sobolev mappings into $\mathbb{H}_{n}$. For more details, see [7]. If $\Omega \subset \mathbb{R}^{m}$ is open and $V$ is a Banach space, then the space of vector valued Sobolev functions $W^{1, p}(\Omega, V)$ can be defined with the notion of Bochner integral and weak derivatives. $W^{1, p}(\Omega, V)$ is a Banach space. Using local coordinate systems (see Section 4 for more details) one can easily extend this definition to the case of mappings from a compact manifold $W^{1, p}(\mathcal{M}, V)$. Any separable metric space and in particular the Heisenberg group $\mathbb{H}_{n}$ admits an isometric embedding into $\ell^{\infty}$ (the Kuratowski embedding). Thus we can assume that $\mathbb{H}_{n} \subset \ell^{\infty}$. Then we define

$$
W^{1, p}\left(\mathcal{M}, \mathbb{H}_{n}\right)=\left\{u \in W^{1, p}\left(\mathcal{M}, \ell^{\infty}\right): u(x) \in \mathbb{H}_{n} \text { a.e. }\right\} \text {. }
$$

The space $W^{1, p}\left(\mathcal{M}, \mathbb{H}_{n}\right)$ is equipped with the norm metric $\rho(u, v)=$ $\|u-v\|_{W^{1, p}}$. The question is whether $\operatorname{Lipschitz} \operatorname{mappings} \operatorname{Lip}\left(\mathcal{M}, \mathbb{H}_{n}\right)$ form a dense subset of $W^{1, p}\left(\mathcal{M}, \mathbb{H}_{n}\right)$, see Theorem 1.9 and the discussion preceding its statement.

The following characterization of bounded Sobolev mappings into $\mathbb{H}_{n}$ was proved in [6], [7, Proposition 6.8].

\section{Proposition 3.1. A bounded function}

$$
f=(z, t)=\left(x_{1}, y_{1}, \ldots, x_{n}, y_{n}, t\right): \Omega \rightarrow \mathbb{H}_{n}
$$

lies in $W^{1, p}\left(\Omega, \mathbb{H}_{n}\right)$ if and only if $f$ is an element of the usual Sobolev space $W^{1, p}\left(\Omega, \mathbb{R}^{2 n+1}\right)$ and satisfies the contact equation

$$
\nabla t=2 \sum_{j=1}^{n}\left(y_{j} \nabla x_{j}-x_{j} \nabla y_{j}\right) \quad \text { a.e. in } \Omega .
$$

Thus the derivative of a Sobolev mapping $f=f\left(u_{1}, \ldots, u_{m}\right)$ maps the tangent space to a horizontal subspace of $\mathbb{H}_{n}$. The length of the gradient $\nabla f$ can be computed with respect to the Euclidean metric $|\nabla f|$ in $\mathbb{R}^{2 n+1}$ or with respect to the sub-Riemannian metric in $\mathbb{H}_{n}$

$$
|\nabla f|_{\mathbb{H}}=\left(\sum_{k=1}^{m}\left|\frac{\partial f}{\partial u_{k}}\right|_{\mathbb{H}}^{2}\right)^{1 / 2}
$$

where $|v|_{\mathbb{H}}$ stands for the length of the horizontal vector with respect to the given metric in the horizontal distribution. If the image of the mapping $f$ is contained in a bounded subset of $\mathbb{H}_{n}$, then both lengths $|\nabla f|$ and $|\nabla f|_{\mathbb{H}}$ are comparable. The following result was proved in [7, Theorem 1.6]. 
Proposition 3.2. Let $\Omega$ be a bounded domain in $\mathbb{R}^{m}$. Suppose that $f_{k}, f \in W^{1, p}\left(\Omega, \mathbb{H}_{n}\right), k=1,2, \ldots, 1 \leq p<\infty, f_{k} \rightarrow f$ in $W^{1, p}\left(\Omega, \mathbb{H}_{n}\right)$. Then

$$
\int_{\left\{f_{k}-f \notin Z\right\}}\left|\nabla f_{k}\right|_{\mathbb{H}}^{p}+|\nabla f|_{\mathbb{H}}^{p} \rightarrow 0 \quad \text { as } k \rightarrow \infty,
$$

where $Z$ denotes the center of $\mathbb{H}_{n}$.

Recall that the center of $\mathbb{H}_{n}$ is the $t$-axis

$$
Z=\left\{(z, t) \in \mathbb{H}_{n}: z=0\right\} .
$$

This result implies that on large sets the difference $f_{k}-f$ must belong to $Z$. This surprisingly strong condition stems from the fact that the Kuratowski embedding of $\mathbb{H}_{n}$ into $\ell^{\infty}$ is highly non-smooth. The identity map id $: \mathbb{H}_{n} \rightarrow \mathbb{R}^{2 n+1}$ is locally Lipschitz and hence if we assume in addition that mappings $f_{k}, f$ are bounded, then $f, f_{k} \in W^{1, p}\left(\Omega, \mathbb{R}^{2 n+1}\right)$. However, it is not obvious that the convergence $f_{k} \rightarrow f$ in $W^{1, p}\left(\Omega, \mathbb{H}_{n}\right)$ implies convergence in $W^{1, p}\left(\Omega, \mathbb{R}^{2 n+1}\right)$, because in general the composition with a Lipschitz function need not be continuous in the Sobolev norm [14, Theorem 1.2]. However the following result is a consequence of Proposition [3.2, see [7, Corollary 1.7].

Corollary 3.3. Let $\mathcal{M}$ be a compact Riemannian manifold. Suppose that $f_{k}, f \in W^{1, p}\left(\mathcal{M}, \mathbb{H}_{n}\right), k=1,2, \ldots$, are uniformly bounded (i.e. the range of all the mappings is contained in a bounded subset of $\left.\mathbb{H}_{n}\right)$. If $f_{k} \rightarrow f$ in $W^{1, p}\left(\mathcal{M}, \mathbb{H}_{n}\right)$, then $f_{k} \rightarrow f$ in $W^{1, p}\left(\mathcal{M}, \mathbb{R}^{2 n+1}\right)$.

We will also need the following fact [7, Lemma 6.5].

Lemma 3.4. Let $f, g \in W^{1, p}\left(\Omega, \mathbb{H}_{n}\right)$. Let $S$ be the set of points $p \in \Omega$ for which $f(p)-g(p) \in Z$. Then $\nabla f=\nabla g$ a.e. in $S$.

\section{Differential forms, Sobolev spaces, and DeRham COHOMOLOGY}

In this section, we recall some notation and properties of differential forms on manifolds, towards the goal of showing that if the DeRham cohomology is zero, then also the $L^{p}$-DeRham cohomology is zero. See Proposition 4.5. This result essentially follows from the $L^{p}$-Hodge decomposition in [20, 24]. 
Before we start, we need to fix some notation. Let $\mathcal{M}$ and $\mathcal{N}$ be $C^{\infty}$-smooth oriented Riemannian manifolds with or without boundary. The volume form will be denoted by dvol. For smooth mappings $f: \mathcal{M} \rightarrow \mathcal{N}$ we let $f^{*}: C^{\infty}\left(\bigwedge^{\ell} \mathcal{N}\right) \rightarrow C^{\infty}\left(\bigwedge^{\ell} \mathcal{M}\right)$ be the pullback of $\ell$-forms. By $d$ we denote the derivative of smooth mappings, $d: C^{\infty}(\mathcal{M}, \mathcal{N}) \rightarrow C^{\infty}(T \mathcal{M}, T \mathcal{N})$, as well as the exterior derivative of $\ell$-forms, $d: C^{\infty}\left(\bigwedge^{\ell} \mathcal{M}\right) \rightarrow C^{\infty}\left(\bigwedge^{\ell+1} \mathcal{M}\right)$. The Hodge operator and the co-differential will be denoted by $* \omega$ and $\delta \omega$, respectively.

Any exterior $\ell$-form $\omega \in \bigwedge^{\ell} \mathcal{M}$ can be expressed in local coordinates $x=\left(x_{1}, \ldots, x_{k}\right): U \subset \mathcal{M} \rightarrow \mathbb{R}^{k}$ by

$$
\omega=\sum_{1 \leq i_{1}<i_{2}<\ldots<i_{\ell} \leq k} \omega_{i_{1}, i_{2}, \ldots i_{\ell}} d x_{i_{1}} \wedge \ldots \wedge d x_{i_{\ell}} \quad \text { in } U .
$$

We only consider local coordinate systems such that $x(U)=\mathbb{B}^{k}(0,1)$ (or $x(U)=\mathbb{B}^{k}(0,1) \cap \mathbb{R}_{+}^{k}$, if we are near the boundary of $\mathcal{M}$ ) and such that they can be smoothly extended to larger domains $V \ni \bar{U}$. This will guarantee boundedness of derivatives of all orders. We say that $\omega \in C^{\infty}\left(\bigwedge^{\ell} \mathcal{M}\right), \operatorname{Lip}\left(\bigwedge^{\ell} \mathcal{M}\right), L^{p}\left(\bigwedge^{\ell} \mathcal{M}\right), W^{1, p}\left(\bigwedge^{\ell} \mathcal{M}\right)$ if the coefficients $\omega_{i_{1}, i_{2}, \ldots i_{\ell}} \circ x^{-1}$ belong to the corresponding space on $\mathbb{B}^{k}(0,1)$ (or $\left.\mathbb{B}^{k}(0,1) \cap \mathbb{R}_{+}^{k}\right)$. Here $W^{1, p}$ is the standard Sobolev space. The norm in $W^{1, p}\left(\bigwedge^{\ell} \mathcal{M}\right)$ is defined as the sum of norms of $\omega_{i_{1}, i_{2}, \ldots i_{\ell}} \circ x^{-1}$ in $W^{1, p}\left(\mathbb{B}^{k}(0,1)\right)$ (or $W^{1, p}\left(\mathbb{B}^{k}(0,1) \cap \mathbb{R}_{+}^{k}\right)$ ) over a finite family of coordinate systems that cover $\mathcal{M}$. A different choice of a family of coordinate systems covering $\mathcal{M}$ will give an equivalent norm. The expression $C_{0}^{\infty}\left(\bigwedge^{\ell} \mathcal{M}\right)$ will stand for smooth $\ell$-forms with compact support. In the case of manifolds with boundary we require the support to be disjoint from the boundary.

We will make frequent use of the identities

$$
f^{*}(\omega \wedge \eta)=f^{*} \omega \wedge f^{*} \eta
$$

and

$$
d\left(f^{*} \eta\right)=f^{*}(d \eta)
$$

Note that (4.1) and (4.2) also hold in a weak sense, in fact, we have

Lemma 4.1. Let $\mathcal{M}$ be a smooth, $k$-dimensional oriented manifold with or without boundary.

(1) If $f \in W_{\mathrm{loc}}^{1,1}\left(\mathcal{M}, \mathbb{R}^{m}\right)$, then (4.1) holds pointwise a.e.

(2) If $f \in W_{\text {loc }}^{1, p}\left(\mathcal{M}, \mathbb{R}^{m}\right), p \geq \ell+1,0 \leq \ell \leq k-1$, and $\eta \in$ $C^{\infty}\left(\bigwedge^{\ell} \mathbb{R}^{m}\right) \cap W^{1, \infty}$ (i.e. $\eta$ and $|\nabla \eta|$ are bounded), then (4.2) 
holds in the weak sense, i.e.

$$
\int_{\mathcal{M}} f^{*} \eta \wedge d \varphi=(-1)^{\ell+1} \int_{\mathcal{M}} f^{*}(d \eta) \wedge \varphi
$$

for all $\varphi \in C_{0}^{\infty}\left(\bigwedge^{k-\ell-1} \mathcal{M}\right)$.

(3) If $\eta \in W_{\mathrm{loc}}^{1, p}\left(\bigwedge^{\ell_{1}} \mathcal{M}\right), \omega \in W_{\mathrm{loc}}^{1, p}\left(\bigwedge^{\ell_{2}} \mathcal{M}\right), \ell_{1}+\ell_{2} \leq k-2, p \geq 2$, then $d(\eta \wedge d \omega)=d \eta \wedge d \omega$ weakly in the sense that

$$
\begin{aligned}
& \qquad \int_{\mathcal{M}} \eta \wedge d \omega \wedge d \varphi=(-1)^{\ell_{1}+\ell_{2}} \int_{\mathcal{M}} d \eta \wedge d \omega \wedge \varphi \\
& \text { for all } \varphi \in C_{0}^{\infty}\left(\wedge^{k-\ell_{1}-\ell_{2}-2} \mathcal{M}\right) .
\end{aligned}
$$

Remark 4.2. In particular (1) and (2) hold under the condition that $f \in W_{\text {loc }}^{1, k}$ and (3) holds under the assumption that $\eta, \omega \in W_{\text {loc }}^{1, k}$. This is what we will need later on.

Proof. (1) is obvious. Regarding (2), observe that $d\left(f^{*} \eta\right)$ is not necessarily well defined in the pointwise sense since $f^{*} \eta$ is only in $L_{\text {loc }}^{p / \ell}$. Thus, we need to interpret the statement in the weak sense. Let $f_{\varepsilon}$ be a smooth approximation of $f$ in $W_{\text {loc }}^{1, p}$. Integration by parts gives

$$
\int_{\mathcal{M}} f_{\varepsilon}^{*} \eta \wedge d \varphi=(-1)^{\ell+1} \int_{\mathcal{M}} f_{\varepsilon}^{*}(d \eta) \wedge \varphi
$$

and the result follows by letting $\varepsilon \rightarrow 0$. The proof of $(3)$ is similar. Let $\omega_{\varepsilon}$ and $\eta_{\varepsilon}$ be smooth approximations of $\omega$ and $\eta$ in $W_{\text {loc }}^{1, p}$. Integration by parts gives

$$
\int_{\mathcal{M}} \eta_{\varepsilon} \wedge d \omega_{\varepsilon} \wedge d \varphi=(-1)^{\ell_{1}+\ell_{2}} \int_{\mathcal{M}} d \eta_{\varepsilon} \wedge d \omega_{\varepsilon} \wedge \varphi
$$

and the result follows by letting $\varepsilon \rightarrow 0$.

Also, we have the following version of the fundamental lemma of the calculus of variations.

Lemma 4.3. Assume $\mathcal{M}$ to be a smooth, $k$-dimensional oriented manifold with or without boundary, and let $\eta \in L_{\mathrm{loc}}^{1}\left(\bigwedge^{\ell} \mathcal{M}\right)$ be such that

$$
\int_{\mathcal{M}} \eta \wedge \varphi=0 \quad \text { for all } \varphi \in C_{0}^{\infty}\left(\bigwedge^{k-\ell} \mathcal{M}\right) \text {. }
$$

Then $\eta=0$ almost everywhere in $\mathcal{M}$. 
Proof. Let $U \subset \mathcal{M}$ be a coordinate patch with coordinate functions $x=\left(x_{1}, \ldots, x_{k}\right): U \rightarrow \mathbb{R}^{k}$. Then

$$
\eta=\sum_{1 \leq i_{1}<\ldots<i_{\ell} \leq k} f_{i_{1} \ldots i_{\ell}} d x_{i_{1}} \wedge \ldots \wedge d x_{i_{\ell}} \quad \text { in } U .
$$

It suffices to show that $f_{i_{1} \ldots i_{\ell}} \circ x^{-1}=0$ a.e. in $x(U)$. Fix $1 \leq i_{1}<\ldots<$ $i_{\ell} \leq k$. For a given $\psi \in C_{0}^{\infty}(x(U))$, let

$$
\varphi:=\psi \circ x|\operatorname{det} D x| d x_{j_{1}} \wedge \ldots \wedge d x_{j_{k-\ell}},
$$

where $\left\{j_{1}, \ldots, j_{k-\ell}\right\}=\{1,2, \ldots, k\} \backslash\left\{i_{1}, \ldots, i_{\ell}\right\}$. Then $\varphi \in$ $C_{0}^{\infty}\left(\bigwedge^{k-\ell} \mathcal{M}\right)$ and consequently

$0=\int_{\mathcal{M}} \eta \wedge \varphi= \pm \int_{\mathcal{M}} f_{i_{1} \ldots i_{\ell}} \cdot \psi \circ x|\operatorname{det} D x| d \mathrm{vol} \equiv \pm \int_{x(U)} f_{i_{1} \ldots i_{\ell}} \circ x^{-1} \cdot \psi$.

Since $f_{i_{1} \ldots i_{\ell}} \circ x^{-1} \in L_{\text {loc }}^{1}(x(U))$, and the test function $\psi \in C_{0}^{\infty}(x(U))$ can be chosen arbitrarily, the classical fundamental lemma of the calculus of variations implies that $f_{i_{1} \ldots i_{\ell}} \circ x^{-1}=0$ almost everywhere in $x(U)$.

We will need the following $L^{p}$-Hodge decomposition [24, Proposition 6.5].

Lemma 4.4 ( $L^{p}$-Hodge Decomposition). Let $\mathcal{M}$ be a smooth, compact, $k$-dimensional oriented manifold without boundary and let $\Omega \subset \mathcal{M}$ be an open subset. Then for any $p \in(1, \infty)$ and any $\ell$-form $\eta \in L^{p}\left(\bigwedge^{\ell} \Omega\right)$, $1 \leq \ell \leq k$ there exist $\omega_{1} \in W^{1, p}\left(\bigwedge^{\ell-1} \Omega\right), \omega_{2} \in W^{1, p}\left(\bigwedge^{\ell+1} \Omega\right)$ such that

$$
\eta=d \omega_{1}+\delta \omega_{2}+h
$$

where $h \in C^{\infty}\left(\bigwedge^{\ell} \Omega\right)$ is closed $d h=0$ and co-closed $\delta h=0$ and hence harmonic.

In the case when $\Omega=\mathcal{M}$ the result was proved in 24, Proposition 6.5$]$ and in the case of a general open set we simply extend $\eta$ to $L^{p}\left(\bigwedge^{\ell} \mathcal{M}\right)$ by zero, apply the Hodge decomposition on $\mathcal{M}$ and restrict all the resulting forms to $\Omega$.

Note that the above result applies to the manifold $\mathcal{M} \times(0,1)$ since it can be isometrically embedded into $\mathcal{M} \times \mathbb{S}^{1}$ as an open set. We will need this special case when we show (Proposition 5.8) that the Hopf invariant is in fact invariant under Lipschitz homotopies.

As an application of the Hodge decomposition we prove that if the DeRham cohomology of an open set $\Omega \subset \mathcal{M}$ is zero, then also the $L^{p}$-DeRham cohomology is zero. More precisely we will show 
Proposition 4.5. Let $\mathcal{M}$ and $\Omega$ be as in Lemma 4.4. Suppose that $H_{D R}^{\ell}(\Omega)=\{0\}$ for some $1 \leq \ell \leq k$, i.e. every smooth closed $\ell$-form on $\Omega$ is exact. Let $\eta \in L^{p}\left(\bigwedge^{\ell} \Omega\right), p \in(1, \infty)$ be weakly closed, i.e.

$$
\int_{\Omega} \eta \wedge d \varphi=0 \quad \text { for all } \varphi \in C_{0}^{\infty}\left(\bigwedge^{k-\ell-1} \Omega\right) \text {. }
$$

Then there exists $\omega \in W_{\mathrm{loc}}^{1, p}\left(\bigwedge^{\ell-1} \Omega\right)$ such that

$$
\eta=d \omega \quad \text { a.e., }
$$

in particular, $\eta$ is exact in the weak sense.

If $\Omega=\mathcal{M}$ is compact without boundary, then $\omega \in W^{1, p}\left(\bigwedge^{\ell-1} \mathcal{M}\right)$ with the estimate

$$
\|\omega\|_{W^{1, p}(\mathcal{M})} \leq C\|\eta\|_{L^{p}(\mathcal{M})},
$$

where the constant $C$ depends only on $\mathcal{M}$, $p$, and the norm in $W^{1, p}(\mathcal{M})$.

Proof. From Lemma 4.4, we obtain $\omega_{1} \in W^{1, p}\left(\bigwedge^{\ell-1} \Omega\right), \omega_{2} \in$ $W^{1, p}\left(\bigwedge^{\ell+1} \Omega\right), h \in C^{\infty}\left(\bigwedge^{\ell} \Omega\right), d h=0, \delta h=0$, such that

$$
\eta=d \omega_{1}+\delta \omega_{2}+h \text {. }
$$

Since $h \in C^{\infty}\left(\Lambda^{\ell} \Omega\right), d h=0$, and $H_{D R}^{\ell}(\Omega)=\{0\}$, there exists $\omega_{3} \in$ $C^{\infty}\left(\bigwedge^{\ell-1} \Omega\right)$ such that $d \omega_{3}=h$. Consequently,

$$
\eta=d\left(\omega_{3}+\omega_{1}\right)+\delta \omega_{2} .
$$

Note that for any $\varphi \in C_{0}^{\infty}\left(\bigwedge^{k-\ell-1} \Omega\right)$, and for any $f \in W_{\text {loc }}^{1, p}\left(\bigwedge^{\ell-1} \Omega\right)$, by approximation

$$
\int_{\Omega} d f \wedge d \varphi=\int_{\Omega} d(f \wedge d \varphi)=0 .
$$

Hence, from (4.4) and (4.6) we infer that for any $\varphi \in C_{0}^{\infty}\left(\bigwedge^{k-\ell-1} \Omega\right)$,

$$
\int_{\Omega} \delta \omega_{2} \wedge d \varphi=\int_{\Omega} d\left(\omega_{3}+\omega_{1}\right) \wedge d \varphi+\int_{\Omega} \delta \omega_{2} \wedge d \varphi=\int_{\Omega} \eta \wedge d \varphi=0,
$$

i.e. $\delta \omega_{2}$ is weakly closed. In particular, for any $\varphi \in C_{0}^{\infty}\left(\bigwedge^{k-\ell} \Omega\right)$

$$
\begin{aligned}
\int_{\Omega} \delta \omega_{2} \wedge(d \delta+\delta d) \varphi & =\int_{\Omega} \delta \omega_{2} \wedge \delta d \varphi \\
& = \pm \int_{\Omega} * d * \omega_{2} \wedge * d * d \varphi \\
& = \pm \int_{\Omega} d * \omega_{2} \wedge d * d \varphi=0
\end{aligned}
$$


where the last equality again follows from approximation and integration by parts just like in (4.7). That is, in the weak sense

$$
\Delta \delta \omega_{2}=0
$$

where $\Delta$ is the Laplace-Beltrami operator. Thus $\delta \omega_{2}$ is actually smooth, see, e.g., [26, Theorem 6.5] or (for the local version) $§ 6.35$ and Exercise 14 on p. 253 of [26]. Since $\delta \omega_{2}$ is weakly closed and smooth, it is closed in the usual sense $d\left(\delta \omega_{2}\right)=0$. Again, $H_{D R}^{\ell}(\Omega)=0$ implies that there is $\omega_{4} \in C^{\infty}\left(\bigwedge^{\ell-1} \Omega\right)$ such that $d \omega_{4}=\delta \omega_{2}$. We have shown that

$$
\eta=d\left(\omega_{3}+\omega_{1}+\omega_{4}\right)
$$

and

$$
\tilde{\omega}:=\omega_{3}+\omega_{1}+\omega_{4} \in W^{1, p}\left(\bigwedge^{\ell-1} \Omega\right)+C^{\infty}\left(\bigwedge^{\ell-1} \Omega\right) \subset W_{\mathrm{loc}}^{1, p}\left(\bigwedge^{\ell-1} \Omega\right) .
$$

If $\Omega \subset \mathcal{M}$ is any open subset, and we do not expect estimate (4.5), we choose $\omega:=\tilde{\omega}$.

Note however, that this choice of $\omega$ is not unique. In fact, setting $\omega:=\tilde{\omega}-\omega_{5}$ for any weakly closed $\omega_{5} \in W^{1, p}$, we have

$$
d \omega=d \tilde{\omega}=\eta \quad \text { a.e. in } \Omega .
$$

If $\Omega=\mathcal{M}$ is compact without boundary then $\tilde{\omega} \in W_{\text {loc }}^{1, p}\left(\bigwedge^{\ell-1} \mathcal{M}\right)=$ $W^{1, p}\left(\bigwedge^{\ell-1} \mathcal{M}\right)$. By [20, Theorem 6.4] there exists a weakly closed form $\omega_{5}$ such that $\omega:=\tilde{\omega}-\omega_{5} \in W^{1, p}\left(\bigwedge^{\ell-1} \mathcal{M}\right)$ satisfies

$$
\|\omega\|_{W^{1, p}}=\left\|\tilde{\omega}-\omega_{5}\right\|_{W^{1, p}} \leq C\|d \tilde{\omega}\|_{L^{p}}=C\|\eta\|_{L^{p}} .
$$

This concludes the proof of Proposition 4.5.

\section{HOPF INVARIANT FOR LOW-RANK MAPPINGS}

Let $\alpha$ be the volume form on $\mathbb{S}^{2 n}$. Then for any smooth mapping $f: \mathbb{S}^{4 n-1} \rightarrow \mathbb{S}^{2 n}$ we have that $d\left(f^{*} \alpha\right)=f^{*}(d \alpha)=0$, so $f^{*} \alpha=d \omega$ for some smooth $2 n-1$ form $\omega$, because $H_{D R}^{2 n}\left(\mathbb{S}^{4 n-1}\right)=\{0\}$. The classical Hopf invariant of $f$ is defined via the Whitehead formula

$$
\mathcal{H} f=\int_{\mathbb{S}^{4 n-1}} \omega \wedge d \omega .
$$

See [5] for details and basic properties.

Hopf [19, Satz II, Satz II'] proved the following important result.

Lemma 5.1. For any $n \in \mathbb{N}$ there exists a smooth map $f: \mathbb{S}^{4 n-1} \rightarrow \mathbb{S}^{2 n}$ such that $\mathcal{H} f \neq 0$. 
In this section we will generalize the Hopf invariant to Lipschitz mappings $f: \mathbb{S}^{4 n-1} \rightarrow \mathbb{R}^{m}, m \geq 2 n+1$, with rank $d f \leq 2 n$ almost everywhere. Let us first give the construction for smooth $f$, rank $d f \leq$ $2 n$. Let $\alpha$ be any smooth $2 n$-form in $\mathbb{R}^{m}$. Since rank $d f \leq 2 n$ and $d \alpha$ is a $(2 n+1)$-form, we have

$$
d\left(f^{*} \alpha\right)=f^{*}(d \alpha)=0
$$

because the determinant of every $(2 n+1)$-dimensional minor of $d f$ has to be zero. Thus there exists a $(2 n-1)$-form $\omega$, such that

$$
d \omega=f^{*} \alpha .
$$

The Hopf invariant of $f$ is defined by

$$
\mathcal{H}_{\alpha} f:=\int_{\mathbb{S}^{4 n-1}} \omega \wedge d \omega .
$$

It depends on $\alpha$, but we will show that $\mathcal{H}_{\alpha} f$ is independent of the particular choice of $\omega$, and that it is actually invariant under Lipschitz homotopies with rank of the derivative less than or equal $2 n$. Obviously, if $f$ is a constant map, then $\mathcal{H}_{\alpha} f=0$. Moreover,

Proposition 5.2. Let $\mathbb{S}^{2 n}$ be isometrically embedded into $\mathbb{R}^{m}, m \geq$ $2 n+1$ and let $\alpha$ be the volume form of $\mathbb{S}^{2 n}$ smoothly extended to $\mathbb{R}^{m}$. Then $\mathcal{H}_{\alpha} f=\mathcal{H} f$ for any smooth $f: \mathbb{S}^{4 n-1} \rightarrow \mathbb{S}^{2 n} \subset \mathbb{R}^{m}$, where $\mathcal{H} f$ is the classical Hopf invariant defined in (5.1). In particular there is a smooth map $f: \mathbb{S}^{4 n-1} \rightarrow \mathbb{R}^{m}$ such that $\mathcal{H}_{\alpha} f \neq 0$.

This is obvious, since rank $d f \leq 2 n$ and $f^{*}\left(\left.\alpha\right|_{\mathbb{S}^{2 n}}\right)=f^{*} \alpha$. The last statement follows from Lemma 5.1 .

Remark 5.3. Observe that the Hopf invariant $\mathcal{H}_{\alpha} f$ is defined for mappings $f: \mathbb{S}^{4 n-1} \rightarrow \mathbb{R}^{m}$. If we denote by $\mathbb{S}^{4 n-1}(r)=r \mathbb{S}^{4 n-1}$ the sphere of radius $r$ centered at the origin, then for mappings $f: \mathbb{S}^{4 n-1}(r) \rightarrow \mathbb{R}^{m}$ we set

$$
\mathcal{H}_{\alpha}\left(\left.f\right|_{\mathbb{S}^{4 n-1}(r)}\right):=\mathcal{H}_{\alpha}\left(\tilde{f}_{r}\right)
$$

where $\tilde{f}_{r}: \mathbb{S}^{4 n-1} \rightarrow \mathbb{R}^{m}$ is defined by $\tilde{f}_{r}(x)=f(r x)$.

5.1. Construction for Lipschitz functions. In order to make our argument precise, we have to ensure that every step above makes sense also for non-smooth Lipschitz mappings. For instance, observe that $f^{*} \alpha$ is only bounded, so one has to interpret $d\left(f^{*} \alpha\right)$ in the weak sense.

This is a non-trivial technicality, as one cannot just approximate $f$ by smooth functions without losing the rank condition, which is essential for the construction of $\omega$. 
First, we confirm that (5.2) holds in a weak sense.

Lemma 5.4. Let $m, k \geq 2 n+1$. Let $\mathcal{M}$ be a smooth $k$-dimensional oriented manifold with or without boundary, and assume that $f: \mathcal{M} \rightarrow$ $\mathbb{R}^{m}$ is a Lipschitz map with rank $d f \leq 2 n$ almost everywhere. Then for any smooth $2 n$-form $\eta \in C^{\infty}\left(\bigwedge^{2 n} \mathbb{R}^{m}\right), f^{*} \eta$ is weakly closed, i.e.

$$
\int_{\mathcal{M}}\left(f^{*} \eta\right) \wedge d \varphi=0 \quad \text { for any } \varphi \in C_{0}^{\infty}\left(\bigwedge^{k-2 n-1} \mathcal{M}\right)
$$

Proof. Let $\varphi \in C_{0}^{\infty}\left(\bigwedge^{k-2 n-1} \mathcal{M}\right)$. Since $f$ is Lipschitz, it is in particular in $W_{\text {loc }}^{1, k}\left(\mathcal{M}, \mathbb{R}^{m}\right)$, so by Lemma 4.1 we have

$$
\int_{\mathcal{M}}\left(f^{*} \eta\right) \wedge d \varphi=-\int_{\mathcal{M}} f^{*}(d \eta) \wedge \varphi=0 .
$$

In Lemma 4.1 we required that $\eta \in C^{\infty} \cap W^{1, \infty}$. However, $W^{1, \infty}$ regularity of $\eta$ is not needed here, because the image of $f$ restricted to the support of $\varphi$ is compact. The last equality in (5.5) follows from the fact that rank $d f \leq 2 n$ a.e. and hence $f^{*}(d \eta)=0$ a.e.

Let $\alpha$ be any smooth $2 n$-form on $\mathbb{R}^{m}, m \geq 2 n+1$ and let $f: \mathbb{S}^{4 n-1} \rightarrow$ $\mathbb{R}^{m}$ be Lipschitz with rank $d f \leq 2 n$ a.e. According to Lemma 5.4, $f^{*} \alpha$ is weakly closed. Since $f^{*} \alpha \in L^{2}\left(\bigwedge^{2 n} \mathbb{S}^{4 n-1}\right)$, Proposition 4.5 and the fact that $H_{D R}^{2 n}\left(\mathbb{S}^{4 n-1}\right)=\{0\}$ imply that there is $\omega \in W^{1,2}\left(\bigwedge^{2 n-1} \mathbb{S}^{4 n-1}\right)$ such that $d \omega=f^{*} \alpha$. Thus, definition (5.4) makes sense also for Lipschitz continuous $f$. Moreover,

Proposition 5.5. Let $\omega_{1}, \omega_{2} \in W^{1, p}\left(\bigwedge^{2 n-1} \mathbb{S}^{4 n-1}\right)$, for some $p \geq 2-\frac{1}{2 n}$, and assume that $d \omega_{1}=d \omega_{2}$ almost everywhere. Then the forms $\omega_{i} \wedge d \omega_{i}$, $i=1,2$ are integrable and

$$
\int_{\mathbb{S}^{4 n-1}} \omega_{1} \wedge d \omega_{1}=\int_{\mathbb{S}^{4 n-1}} \omega_{2} \wedge d \omega_{2}
$$

In particular, for any Lipschitz map $f: \mathbb{S}^{4 n-1} \rightarrow \mathbb{R}^{m}$ with rank $d f \leq$ $2 n$ a.e., definition (5.4) of $\mathcal{H}_{\alpha} f$ is independent of the choice of $\omega \in$ $W^{1, p}\left(\bigwedge^{2 n-1} \mathbb{S}^{4 n-1}\right)$ with $d \omega=f^{*} \alpha$.

This result easily follows from a slightly more general fact.

Proposition 5.6. If $\omega, \nu \in W^{1, p}\left(\bigwedge^{2 n-1} \mathbb{S}^{4 n-1}\right)$, for some $p \geq 2-\frac{1}{2 n}$, then $\omega \wedge \nu \in W^{1,1}\left(\bigwedge^{4 n-2} \mathbb{S}^{4 n-1}\right), d \omega \wedge \nu, \omega \wedge d \nu \in L^{1}\left(\bigwedge^{4 n-1} \mathbb{S}^{4 n-1}\right)$, $d(\omega \wedge \nu)=d \omega \wedge \nu-\omega \wedge d \nu$ a.e. and

$$
\int_{\mathbb{S}^{4 n-1}} d \omega \wedge \nu=\int_{\mathbb{S}^{4 n-1}} \omega \wedge d \nu
$$


Assuming for a moment the validity of Proposition 5.6, we show how to complete the proof of Proposition 5.5. We have

$$
\int_{\mathbb{S}^{4 n-1}} d \omega_{1} \wedge\left(\omega_{1}-\omega_{2}\right)=\int_{\mathbb{S}^{4 n-1}} \omega_{1} \wedge d\left(\omega_{1}-\omega_{2}\right)=0,
$$

and hence,

$$
\int_{\mathbb{S}^{4 n-1}} d \omega_{1} \wedge \omega_{1}=\int_{\mathbb{S}^{4 n-1}} d \omega_{1} \wedge \omega_{2}=\int_{\mathbb{S}^{4 n-1}} d \omega_{2} \wedge \omega_{2} .
$$

This proves Proposition 5.5.

Proof of Proposition 5.6. We will need the following auxiliary result.

Lemma 5.7. If $f, g \in W^{1, \frac{2 k}{k+1}}\left(\mathbb{R}^{k}\right)$, then $f g \in W^{1,1}\left(\mathbb{R}^{k}\right)$.

Proof. Let $p=2 k /(k+1)$. If $k \geq 2$, an easy calculation shows that the Sobolev exponent satisfies $p^{*}=p /(p-1)$ and hence by Hölder's inequality and the Sobolev embedding theorem $f g \in L^{1}$. This is also true for $k=1$ since $W^{1,1}(\mathbb{R}) \subset L^{\infty}$. Sobolev functions are absolutely continuous on almost every line [9, Section 4.9]. Since the product of absolutely continuous functions is absolutely continuous, $f g$ is also absolutely continuous on almost every line. Hence we can compute partial derivatives

$$
\frac{\partial}{\partial x_{i}}(f g)=\frac{\partial f}{\partial x_{i}} g+f \frac{\partial g}{\partial x_{i}}
$$

Again, since $p^{*}=p /(p-1)$ we conclude that $\partial(f g) / \partial x_{i} \in L^{1}$ for $i=1,2, \ldots, k$. The characterization of $W^{1,1}$ by absolute continuity on lines [9, Section 4.9] implies that $f g \in W^{1,1}\left(\mathbb{R}^{k}\right)$.

Let $\omega, \nu \in W^{1, p}\left(\bigwedge^{2 n-1} \mathbb{S}^{4 n-1}\right)$. We can assume that $p=2-\frac{1}{2 n}$. If $k=4 n-1$, then

$$
\frac{2 k}{k+1}=2-\frac{1}{2 n}
$$

Thus applying the lemma to representations of $\omega$ and $\nu$ in local coordinates we obtain that $\omega \wedge \nu \in W^{1,1}\left(\bigwedge^{4 n-2} \mathbb{S}^{4 n-1}\right)$. The product rule (5.6) yields

$$
d(\omega \wedge \nu)=d \omega \wedge \nu-\omega \wedge d \nu \quad \text { a.e. }
$$

It follows from the Sobolev embedding theorem that $\omega, \nu \in$ $L^{\frac{p}{p-1}}\left(\bigwedge^{2 n-1} \mathbb{S}^{4 n-1}\right)$. This and the Hölder inequality imply

$$
d \omega \wedge \nu, \omega \wedge d \nu \in L^{1}\left(\bigwedge^{4 n-1} \mathbb{S}^{4 n-1}\right)
$$


Finally, integrating (5.7) we get

$$
\int_{\mathbb{S}^{4 n-1}} d \omega \wedge \nu-\int_{\mathbb{S}^{4 n-1}} \omega \wedge d \nu=\int_{\mathbb{S}^{4 n-1}} d(\omega \wedge \nu)=0 .
$$

The last equality uses Stokes' theorem, which holds by approximating $\omega \wedge \nu \in W^{1,1}\left(\bigwedge^{4 n-2} \mathbb{S}^{4 n-1}\right)$ by smooth $(4 n-2)$-forms.

Next, we show that $\mathcal{H}_{\alpha} f$ is invariant under Lipschitz homotopies of rank at most $2 n$.

Proposition 5.8. Let $f, g: \mathbb{S}^{4 n-1} \rightarrow \mathbb{R}^{m}$ be two Lipschitz maps of rank at most $2 n$ and assume that there is a Lipschitz homotopy

$$
H:[0,1] \times \mathbb{S}^{4 n-1} \rightarrow \mathbb{R}^{m}, \quad H(0, \cdot)=f(\cdot), H(1, \cdot)=g(\cdot),
$$

such that rank $d H \leq 2 n$ a.e. Then

$$
\mathcal{H}_{\alpha} f=\mathcal{H}_{\alpha} g \text {. }
$$

Proof. We adapt the argument from [5, Proposition 17.22.(c)]. However, since we are dealing with non-smooth mappings we have to be very careful. We may assume that $H:[0,1] \times \mathbb{S}^{4 n-1} \rightarrow \mathbb{R}^{m}$ is constant in $t$ for $0 \leq t \leq 1 / 4$ and $3 / 4 \leq t \leq 1$. If not, we take a Lipschitz function $s(t):[0,1] \rightarrow[0,1]$ such that

$$
s(t):= \begin{cases}0 & 0 \leq t \leq \frac{1}{4} \\ 1 & \frac{3}{4} \leq t \leq 1\end{cases}
$$

and consider $H(s(t), x)$ instead of $H(t, x)$, which is still Lipschitz, and also satisfies the rank condition. We have

$$
H^{*} \alpha \in L^{\infty}\left(\bigwedge^{2 n}\left((0,1) \times \mathbb{S}^{4 n-1}\right)\right)
$$

Since $\mathbb{S}^{4 n-1}$ is a deformation retract of $(0,1) \times \mathbb{S}^{4 n-1}$ we conclude that $H_{D R}^{2 n}\left((0,1) \times \mathbb{S}^{4 n-1}\right)=H_{D R}^{2 n}\left(\mathbb{S}^{4 n-1}\right)=\{0\},[5$, Corollary 4.1.2.2]. Now from the fact that rank $d H \leq 2 n$ and from Lemma 5.4 we infer that $H^{*} \alpha$ is weakly closed. Since $(0,1) \times \mathbb{S}^{4 n-1}$ can be isometrically embedded into the compact manifold $\mathbb{S}^{1} \times \mathbb{S}^{4 n-1}$ as an open set by Proposition 4.5 there is $\omega \in W_{\text {loc }}^{1,2}\left(\bigwedge^{2 n-1}(0,1) \times \mathbb{S}^{4 n-1}\right)$ such that

$$
d \omega=H^{*} \alpha \quad \text { a.e. }
$$

Denote by

$$
\imath_{t}: \mathbb{S}^{4 n-1} \rightarrow\{t\} \times \mathbb{S}^{4 n-1} \subset(0,1) \times \mathbb{S}^{4 n-1} .
$$

the canonical embedding of the sphere by the identity. From the Rademacher and Fubini theorems it follows that for almost every 
$t \in(0,1), H$ is differentiable at almost all points of the sphere $\{t\} \times \mathbb{S}^{4 n-1}$. Thus the chain rule implies that

$$
\left(H \circ \imath_{t}\right)^{*} \alpha=\imath_{t}^{*} H^{*} \alpha \quad \text { a.e. in } \mathbb{S}^{4 n-1}
$$

Note also that $\omega_{t}:=\imath_{t}^{*} \omega$ is defined a.e. on $\mathbb{S}^{4 n-1}$ for almost all $t \in(0,1)$. Approximate $\omega$ by

$$
\omega^{\varepsilon} \in C^{\infty}\left(\bigwedge^{2 n-1}(0,1) \times \mathbb{S}^{4 n-1}\right) \quad \text { in } \quad W_{\mathrm{loc}}^{1,2}\left(\bigwedge^{2 n-1}(0,1) \times \mathbb{S}^{4 n-1}\right)
$$

It follows from Fubini's theorem (cf. [13, p. 189]) that there is a sequence $\varepsilon_{i} \rightarrow 0$ such that

$$
\omega_{t}^{\varepsilon_{i}}:=\imath_{t}^{*} \omega^{\varepsilon_{i}} \rightarrow \imath_{t}^{*} \omega=\omega_{t} \quad \text { in } \quad W^{1,2}\left(\bigwedge^{2 n-1} \mathbb{S}^{4 n-1}\right)
$$

and

$$
\imath_{t}^{*} d \omega^{\varepsilon_{i}} \rightarrow \imath_{t}^{*} d \omega \quad \text { in } \quad L^{2}\left(\bigwedge^{2 n} \mathbb{S}^{4 n-1}\right)
$$

for almost all $t \in(0,1)$. Since

$$
\imath_{t}^{*} d \omega^{\varepsilon_{i}}=d \imath_{t}^{*} \omega^{\varepsilon_{i}} \rightarrow d \omega_{t} \quad \text { in } L^{2}
$$

we conclude that

$$
d \omega_{t}=\imath_{t}^{*} d \omega \quad \text { a.e. on } \mathbb{S}^{4 n-1} \text { for a.e. } t \in(0,1) .
$$

Fix $t_{0} \in(0,1 / 4)$ and $t_{1} \in(3 / 4,1)$ such that (5.8), (5.9) and (5.10) are satisfied. We have

$$
d \omega_{t_{0}}=\imath_{t_{0}}^{*} d \omega=\imath_{t_{0}}^{*} H^{*} \alpha=\left(H \circ \imath_{t_{0}}\right)^{*} \alpha=f^{*} \alpha \quad \text { a.e. in } \mathbb{S}^{4 n-1}
$$

Similarly

$$
d \omega_{t_{1}}=g^{*} \alpha \quad \text { a.e. in } \mathbb{S}^{4 n-1}
$$


Hence

$$
\begin{aligned}
\mathcal{H}_{\alpha} f-\mathcal{H}_{\alpha} g & =\int_{\mathbb{S}^{4 n-1}} \omega_{t_{0}} \wedge d \omega_{t_{0}}-\int_{\mathbb{S}^{4 n-1}} \omega_{t_{1}} \wedge d \omega_{t_{1}} \\
& =\lim _{i \rightarrow \infty}\left(\int_{\mathbb{S}^{4 n-1}} \omega_{t_{0}}^{\varepsilon_{i}} \wedge d \omega_{t_{0}}^{\varepsilon_{i}}-\int_{\mathbb{S}^{4 n-1}} \omega_{t_{1}}^{\varepsilon_{i}} \wedge d \omega_{t_{1}}^{\varepsilon_{i}}\right) \\
& =\lim _{i \rightarrow \infty} \int_{\partial\left(\left(t_{0}, t_{1}\right) \times \mathbb{S}^{4 n-1}\right)} \omega^{\varepsilon_{i}} \wedge d \omega^{\varepsilon_{i}} \\
& =\lim _{i \rightarrow \infty} \int_{\left(t_{0}, t_{1}\right) \times \mathbb{S}^{4 n-1}} d\left(\omega^{\varepsilon_{i}} \wedge d \omega^{\varepsilon_{i}}\right) \\
& =\lim _{i \rightarrow \infty} \int_{\left(t_{0}, t_{1}\right) \times \mathbb{S}^{4 n-1}} d \omega^{\varepsilon_{i}} \wedge d \omega^{\varepsilon_{i}} \\
& =\int_{\left(t_{0}, t_{1}\right) \times \mathbb{S}^{4 n-1}} d \omega \wedge d \omega \\
& =\int_{\left(t_{0}, t_{1}\right) \times \mathbb{S}^{4 n-1}} H^{*} \alpha \wedge H^{*} \alpha \\
& =\int_{\left(t_{0}, t_{1}\right) \times \mathbb{S}^{4 n-1}} H^{*}(\alpha \wedge \alpha)=0 .
\end{aligned}
$$

The last equality follows from the fact that rank $d H \leq 2 n$ a.e. and $\alpha \wedge \alpha$ is a $4 n$-form, so $H^{*}(\alpha \wedge \alpha)=0$ a.e.

We will also need the following convergence result.

Proposition 5.9. Let $g_{k}, g \in \operatorname{Lip}\left(\mathbb{S}^{4 n-1}, \mathbb{R}^{m}\right)$ be Lipschitz mappings with rank $d g_{k}$, rank $d g \leq 2 n$ almost everywhere and such that for a given $\alpha \in C^{\infty}\left(\bigwedge^{2 n} \mathbb{R}^{m}\right)$

$$
\lim _{k \rightarrow \infty}\left\|g_{k}^{*} \alpha-g^{*} \alpha\right\|_{L^{p}\left(\bigwedge^{2 n} \mathbb{S}^{4 n-1}\right)}=0
$$

for some $p \geq 2-\frac{1}{2 n}$. Then

$$
\lim _{k \rightarrow \infty} \mathcal{H}_{\alpha} g_{k}=\mathcal{H}_{\alpha} g
$$

Proof. We can assume that $p=2-\frac{1}{2 n}$. According to Lemma 5.4 the forms $g^{*} \alpha$ and $g_{k}^{*} \alpha$ are weakly closed. Hence from Proposition 4.5 there exist $\omega, \omega_{k} \in W^{1, p}\left(\bigwedge^{2 n-1} \mathbb{S}^{4 n-1}\right)$ with $d \omega=g^{*} \alpha, d \omega_{k}=g_{k}^{*} \alpha$, and such that

$$
\|\omega\|_{L^{\frac{p}{p-1}}} \leq C\|\omega\|_{W^{1, p}} \leq C^{\prime}\left\|g^{*} \alpha\right\|_{L^{p}}
$$

and similarly

$$
\left\|\omega_{k}\right\|_{L^{\frac{p}{p-1}}} \leq C^{\prime}\left\|g_{k}^{*} \alpha\right\|_{L^{p}}
$$


We used here the Sobolev inequality and the fact that $p^{*}=\frac{p}{p-1}$. In view of Proposition 5.5.

$$
\mathcal{H}_{\alpha} g=\int_{\mathbb{S}^{4 n-1}} \omega \wedge d \omega, \quad \mathcal{H}_{\alpha} g_{k}=\int_{\mathbb{S}^{4 n-1}} \omega_{k} \wedge d \omega_{k}
$$

Hence

$$
\begin{aligned}
\mathcal{H}_{\alpha} g_{k}-\mathcal{H}_{\alpha} g & =\int_{\mathbb{S}^{4 n-1}} \omega_{k} \wedge d \omega_{k}-\omega \wedge d \omega \\
& =\int_{\mathbb{S}^{4 n-1}} \omega_{k} \wedge\left(d \omega_{k}-d \omega\right)+\left(\omega_{k}-\omega\right) \wedge d \omega \\
& =\int_{\mathbb{S}^{4 n-1}} \omega_{k} \wedge\left(d \omega_{k}-d \omega\right)+d\left(\omega_{k}-\omega\right) \wedge \omega \\
& \leq C\left(\left\|\omega_{k}\right\|_{L^{\frac{p}{p-1}}}+\|\omega\|_{L^{\frac{p}{p-1}}}\right)\left\|g^{*} \alpha-g_{k}^{*} \alpha\right\|_{L^{p}} \\
& \leq C\left(\left\|g_{k}^{*} \alpha\right\|_{L^{p}}+\left\|g^{*} \alpha\right\|_{L^{p}}\right)\left\|g^{*} \alpha-g_{k}^{*} \alpha\right\|_{L^{p}} \stackrel{k \rightarrow \infty}{\longrightarrow} 0 .
\end{aligned}
$$

The third-last equality follows from Proposition 5.6.

\section{Proof of Theorem 1.7}

The case of $\pi_{n}\left(\mathbb{S}^{n}\right)$ having already been proved in Section 1, it remains to show that the homotopy group $\pi_{4 n-1}\left(\mathbb{S}^{2 n}\right)$ is rank-essential for $n \in \mathbb{N}$. Let $f: \mathbb{S}^{4 n-1} \rightarrow \mathbb{S}^{2 n} \subset \mathbb{R}^{2 n+1}$ be the mapping, and $\alpha$ the $2 n$-form on $\mathbb{R}^{2 n+1}$ such that

$$
\mathcal{H}_{\alpha} f \neq 0 \text {. }
$$

See Proposition 5.2. Assume by contradiction that $\pi_{4 n-1}\left(\mathbb{S}^{2 n}\right)$ is not rank-essential. Hence there exists a Lipschitz extension $F: \mathbb{B}^{4 n} \rightarrow$ $\mathbb{R}^{2 n+1}$ such that rank $d F \leq 2 n$ almost everywhere in $\mathbb{B}^{4 n}$. Define the homotopy

$$
H(t, \theta):[0,1] \times \mathbb{S}^{4 n-1} \rightarrow \mathbb{R}^{2 n+1}
$$

between $f=H(1, \cdot)$ and a constant map $g=H(0, \cdot)$ via

$$
H(t, \theta):=F(t \theta) .
$$

This homotopy $H$ is clearly Lipschitz, with $\operatorname{rank} d H \leq 2 n$. Obviously, rank $d f$ and rank $d g$ do not exceed $2 n$. Then, since the Hopf invariant $\mathcal{H}_{\alpha} f$ does not change under Lipschitz rank $2 n$-homotopies, see Proposition 5.8 ,

$$
\mathcal{H}_{\alpha} f=\mathcal{H}_{\alpha} g=0
$$

which contradicts (6.1). The proof is complete. 


\section{Proof of Theorem 1.9}

The proof is similar to that of Theorem 1.2(a) and Proposition 1.3 in [7.

Assume first that $\mathcal{M}=\mathbb{B}^{4 n}$. Let $\phi: \mathbb{S}^{2 n} \rightarrow \mathbb{H}_{2 n}$ be a bi-Lipschitz map, which is a smooth embedding as a map from $\mathbb{S}^{2 n}$ to $\mathbb{R}^{4 n+1}$, see Proposition 1.2. Let $f_{0} \in C^{\infty}\left(\mathbb{S}^{4 n-1}, \mathbb{S}^{2 n}\right)$ be the Hopf map from Lemma 5.1 such that

$$
\mathcal{H} f_{0} \neq 0 \text {. }
$$

It easily follows from Proposition 3.1 that

$$
f(x):=\phi \circ f_{0}\left(\frac{x}{|x|}\right) \in W^{1, p}\left(\mathbb{B}^{4 n}, \mathbb{H}_{2 n}\right), \quad \text { for all } 1 \leq p<4 n .
$$

We will prove that $f$ cannot be approximated in $W^{1, p}\left(\mathbb{B}^{4 n}, \mathbb{H}_{2 n}\right)$ by Lipschitz mappings $\operatorname{Lip}\left(\mathbb{B}^{4 n}, \mathbb{H}_{2 n}\right)$ when $4 n-1 \leq p<4 n$. Suppose to the contrary that there is a sequence $g_{k} \in \operatorname{Lip}\left(\mathbb{B}^{4 n}, \mathbb{H}_{2 n}\right)$ such that

$$
g_{k} \rightarrow f \quad \text { in } W^{1, p}\left(\mathbb{B}^{4 n}, \mathbb{H}_{2 n}\right) .
$$

Note that by Proposition 1.1 both rank $d g_{k}$ and rank $d f$ do not exceed $2 n$. Formally, $f$ is not Lipschitz, but it is locally Lipschitz away from the singularity at the origin and hence Proposition 1.1 applies to $f$ as well.

Choose $\alpha \in C_{0}^{\infty}\left(\bigwedge^{2 n} \mathbb{R}^{4 n+1}\right)$ to be a smooth extension of the pushforward $\phi_{*} d$ vol $_{\mathbb{S}^{2 n}}$. Recalling our definition of the Hopf invariant of mappings whose domains are scaled spheres $\mathbb{S}^{4 n-1}(r)$, see Remark 5.3,

$$
\mathcal{H}_{\alpha}\left(\left.f\right|_{\mathbb{S}^{4 n-1}(r)}\right)=\mathcal{H}\left(f_{0}\right) \neq 0 \quad \text { for all } r \in(0,1) .
$$

On the other hand, $g_{k} \in \operatorname{Lip}\left(\mathbb{B}^{4 n}, \mathbb{H}_{2 n}\right)$, and hence $\left.g_{k}\right|_{\mathbb{S}^{4 n-1}(r)}$ as a mapping to $\mathbb{R}^{4 n+1}$ is Lipschitz homotopic to a constant map with the homotopy satisfying the rank condition rank $d H \leq 2 n$ a.e. (see Proposition 1.1). Thus Proposition 5.8 yields

$$
\mathcal{H}_{\alpha}\left(\left.g_{k}\right|_{\mathbb{S}^{4 n-1}(r)}\right)=0 \quad \text { for all } k \text { and all } r \in(0,1) .
$$

We are now going to show that (7.1) and (7.2) contradict each other.

Since the mappings $g_{k}$ are not necessarily uniformly bounded we cannot claim that $g_{k} \rightarrow f$ in $W^{1, p}\left(\mathbb{B}^{4 n}, \mathbb{R}^{4 n+1}\right)$, see Corollary 3.3. In particular we cannot claim that $\nabla g_{k} \rightarrow \nabla f$ in $L^{p}\left(\mathbb{B}^{4 n}\right)$. Nevertheless 
we can assume upon passing to a subsequence that $g_{k} \rightarrow f$ a.e. in $\mathbb{B}^{4 n}$. We will construct sets $E_{k}$ such that

$$
\chi_{E_{k}} \nabla g_{k} \rightarrow \nabla f \quad \text { in } L^{p}\left(\mathbb{B}^{4 n}\right) .
$$

Let $K=\operatorname{supp} \alpha$, let

$$
S_{k}=\left\{x \in \mathbb{B}^{4 n}: g_{k}(x)-f(x) \in Z\right\},
$$

where $Z$ is the center of $\mathbb{H}_{2 n}$ defined in (3.1), and let

$$
E_{k}=S_{k} \cup g_{k}^{-1}(K) .
$$

We claim that (17.3) is true. According to Lemma 3.4, $\nabla g_{k}=\nabla f$ a.e. in $S_{k}$ and hence

$$
\int_{S_{k}}\left|\nabla f-\nabla g_{k}\right|^{p}=0
$$

Since the mappings $f$ and $\left.g_{k}\right|_{g_{k}^{-1}(K)}$ are uniformly bounded, the Euclidean lengths $|\nabla f|$ and $\left|\chi_{E_{k}} \nabla g_{k}\right|$ are comparable to the Heisenberg lengths $|\nabla f|_{\mathbb{H}}$ and $\left|\chi_{E_{k}} \nabla g_{k}\right|_{\mathbb{H}}$ respectively on the set $\mathbb{B}^{4 n} \backslash S_{k}$. Thus Proposition 3.2 yields

$$
\int_{\mathbb{B}^{4 n} \backslash S_{k}}|\nabla f|^{p}+\left|\chi_{E_{k}} \nabla g_{k}\right|^{p} \leq C \int_{\mathbb{B}^{4 n} \backslash S_{k}}|\nabla f|_{\mathbb{H}}^{p}+\left|\chi_{E_{k}} \nabla g_{k}\right|_{\mathbb{H}}^{p} \rightarrow 0 .
$$

Hence

$$
\begin{aligned}
& \int_{\mathbb{B}^{4 n}}\left|\nabla f-\chi_{E_{k}} \nabla g_{k}\right|^{p} \\
& \quad \leq C\left(\int_{S_{k}}\left|\nabla f-\nabla g_{k}\right|^{p}+\int_{\mathbb{B}^{4 n} \backslash S_{k}}|\nabla f|^{p}+\left|\chi_{E_{k}} \nabla g_{k}\right|^{p}\right) \rightarrow 0 .
\end{aligned}
$$

Now it follows from Fubini's theorem that, up to a subsequence which we again denote by $g_{k}$,

$$
\left.\left.\chi_{E_{k}} \nabla g_{k}\right|_{\mathbb{S}^{4 n-1}(r)} \stackrel{k \rightarrow \infty}{\longrightarrow} \nabla f\right|_{\mathbb{S}^{4 n-1}(r)} \quad \text { in } L^{p}\left(\mathbb{S}^{4 n-1}(r)\right)
$$

for almost any $r \in(0,1)$. This and the almost everywhere convergence $g_{k} \rightarrow f$ implies that

$$
\chi_{E_{k}}\left(\left.g_{k}\right|_{\mathbb{S}^{4 n-1}(r)}\right)^{*} \alpha \rightarrow\left(\left.f\right|_{\mathbb{S}^{4 n-1}(r)}\right)^{*} \alpha \quad \text { in } L^{p / 2 n}\left(\bigwedge^{2 n} \mathbb{S}^{4 n-1}(r)\right)
$$

for almost all $r \in(0,1)$. On the other hand, since $K=\operatorname{supp} \alpha, g_{k}^{*} \alpha=0$ a.e. in $\mathbb{B}^{4 n} \backslash g_{k}^{-1}(K)$ and hence $g_{k}^{*} \alpha=0$ a.e. in $\mathbb{B}^{4 n} \backslash E_{k}$. Accordingly,

$$
\left(\left.g_{k}\right|_{\mathbb{S}^{4 n-1}(r)}\right)^{*} \alpha=\chi_{E_{k}}\left(\left.g_{k}\right|_{\mathbb{S}^{4 n-1}(r)}\right)^{*} \alpha \quad \text { for a.e. } r \in(0,1),
$$


which, in conjunction with (7.4), yields

$$
\lim _{k \rightarrow \infty}\left\|\left(\left.g_{k}\right|_{\mathbb{S}^{4 n-1}(r)}\right)^{*} \alpha-\left(\left.f\right|_{\mathbb{S}^{4 n-1}(r)}\right)^{*} \alpha\right\|_{L^{p / 2 n}\left(\bigwedge^{2 n} \mathbb{S}^{4 n-1}(r)\right)}=0 .
$$

This, (7.1), and Proposition 5.9 imply that for $p \geq 4 n-1$ and almost all $r \in(0,1)$, we have

$$
\lim _{k \rightarrow \infty} \mathcal{H}_{\alpha}\left(\left.g_{k}\right|_{\mathbb{S}^{4 n-1}(r)}\right)=\mathcal{H}_{\alpha}\left(\left.f\right|_{\mathbb{S}^{4 n-1}(r)}\right) \neq 0 .
$$

This conclusion contradicts (7.2).

If $\mathcal{M}$ is a general manifold of dimension $\operatorname{dim} \mathcal{M} \geq 4 n$, then the result follows from the case $\mathbb{B}^{4 n}$ by a simple surgery as in the proof of Theorem 1.2 in [7]. We simply construct a mapping $f \in W^{1, p}\left(\mathcal{M}, \mathbb{H}_{2 n}\right)$ such that on a family of $4 n$ dimensional slices in $\mathcal{M}$ it coincides with the mapping constructed above. By using the Fubini theorem one easily arrives at a contradiction by employing the case of $\mathbb{B}^{4 n}$.

\section{REFERENCES}

[1] Balogh, Z., Fässler, K. S., Rectifiability and Lipschitz extensions into the Heisenberg group. Math. Z. 263 (2009), 673-683.

[2] Bates, S. M.: Toward a precise smoothness hypothesis in Sard's theorem. Proc. Amer. Math. Soc. 117 (1993), 279-283.

[3] Bethuel, F., The approximation problem for Sobolev maps between two manifolds. Acta Math. 167 (1991), 153-206.

[4] Bethuel, F., Zheng, X. M., Density of smooth functions between two manifolds in Sobolev spaces. J. Funct. Anal. 80 (1988), 60-75.

[5] Bott, R., And Tu, L.-W.: Differential forms in algebraic topology, volume 82 of Graduate Texts in Mathematics. Springer-Verlag, New York, 1982.

[6] Capogna, L., Lin, F.-H., Legendrian energy minimizers. I. Heisenberg group target. Calc. Var. Partial Differential Equations 12 (2001), 145-171.

[7] DeJarnette, N., HajŁasz, P., Lukyanenko, A., Tyson, J.: On the lack of density of Lipschitz mappings in Sobolev spaces with Heisenberg target. Preprint, arXiv:1109.4641, 2011.

[8] Ekholm, T., Etnyre, J., Sullivan, M., Non-isotopic Legendrian submanifolds in $\mathbb{R}^{2 n+1}$. J. Differential Geom. 71 (2005), 85-128.

[9] Evans, L.C. And GariePY, R.F.: Measure theory and fine properties of functions, Studies in Advanced Mathematics. CRC Press, Boca Raton, FL, 1992.

[10] Federer, H.: Geometric measure theory, volume 153 of Grundlehren der mathematischen Wissenschaften. Springer-Verlag, New York, 1969.

[11] Félix, Y., Halperin, S., Thomas, J.-C.: Rational homotopy theory. Graduate Texts in Mathematics, 205. Springer-Verlag, New York, 2001.

[12] HajŁasz, P., Density of Lipschitz mappings in the class of Sobolev mappings between metric spaces. Math. Ann. 343 (2009), 801-823. 
[13] HajŁasz, P., Sobolev mappings between manifolds and metric spaces. In: Sobolev spaces in mathematics. I, pp. 185-222, Int. Math. Ser. (N.Y.), 8, Springer, New York, 2009.

[14] HajŁasz, P., Sobolev mappings: Lipschitz density is not a bi-Lipschitz invariant of the target. Geom. Funct. Anal. 17 (2007), 435-467.

[15] HajŁasz, P., Approximation of Sobolev mappings. Nonlinear Anal. 22 (1994), 1579-1591.

[16] Hajeasz, P., Koskela, P., Sobolev met Poincaré. Memoirs Amer. Math. Soc. 688 (2000), 1-101.

[17] HajŁasz, P., Schikorra, A.: Lipschitz homotopy and density of Lipschitz mappings in Sobolev spaces. Preprint, arXiv:1306.6502, 2013.

[18] Hang, F., Lin, F., Topology of Sobolev mappings II. Acta Math. 191 (2003), $55-107$.

[19] Hopf, H.: Über die Abbildungen von Sphären auf Sphären niedrigerer Dimensionen. (German) Fundamenta Math. 25 (1935), 427-440.

[20] Iwaniec, T., Scott, C. And Stroffolini, B.: Nonlinear Hodge theory on manifolds with boundary. Ann. Mat. Pura Appl. (4), 177 (1999), 37-115.

[21] Kaufman, R.: A singular map of a cube onto a square. J. Differential Geom. 14 (1979), 593-594 (1981).

[22] Mattila, P.: Geometry of sets and measures in Euclidean spaces. Fractals and rectifiability. Cambridge Studies in Advanced Mathematics, 44. Cambridge University Press, Cambridge, 1995.

[23] Rigot, S., Wenger, S.: Lipschitz non-extension theorems into jet space Carnot groups. Int. Math. Res. Not. IMRN 2010, no. 18, 3633-3648.

[24] Sсотт, C.: $L^{p}$ theory of differential forms on manifolds. Trans. Amer. Math. Soc., 347 (1995), 2075-2096.

[25] Serre, J.-P.: Groupes d'homotopie et classes de groupes abéliens. Ann. of Math. 58 (1953), 258-294.

[26] Warner, F., Foundations of Differentiable Manifolds and Lie Groups. Graduate Texts in Mathematics, 94. Springer-Verlag, New York, 1983.

[27] Wenger, S., Young, R., Lipschitz extensions into jet space Carnot groups. Math. Res. Letters 17 (2010), 1137-1149.

[28] Wenger, S., Young, R., Lipschitz homotopy groups of the Heisenberg groups. Preprint, arXiv:1210.6943, 2012.

Piotr Hajeasz, Department of Mathematics, University of Pittsburgh, 301 Thackeray Hall, Pittsburgh, PA 15260, USA, hajlasz@pitt.edu

Armin Schikorra, Max-Planck Institut MiS Leipzig, Inselstr. 22, 04103 LeIPZIG, GERMANY, armin.schikorra@mis.mpg.de

Jeremy T. Tyson, Department of Mathematics, University of Illinois at Urbana-Champaign, 1409 West Green Street, Urbana, IL 61801, USA, tyson@math.uiuc.edu 